\title{
Subharmonic instabilities in resonant interactions with bichromatic fields
}

\author{
Karl Koch, Brian J. Oliver, Stephen H. Chakmakjian, and C. R. Stroud, Jr. \\ The Institute of Optics, University of Rochester, Rochester, New York \\ Lloyd W. Hillman \\ School of Electrical Engineering, Cornell University, Ithaca, New York 14853
}

Received July 12, 1988; accepted October 3, 1988

\begin{abstract}
We consider the interaction of two $100 \%$ amplitude-modulated fields with a two-level system. The analysis includes a discussion of the stability of an amplitude-modulated laser field to the growth of subharmonic amplitude-
modulated fields.
\end{abstract}

\section{INTRODUCTION}

Recently a great deal of interest has focused on instabilities in laser systems. ${ }^{1,2}$ Motivation of this interest is provided by a desire to understand the complicated dynamics of nonlinear systems. Optical systems provide a convenient ground for rigorously examining these dynamics. The laser is a specific example of a rigorously describable optical system containing the nonlinear interaction of light with matter. Subharmonic instabilities have been observed in a number of different lasers. ${ }^{3-5}$ Subharmonic instabilities have become especially interesting since a sequence of period doublings has been identified as a possible route to chaos.

Instabilities in lasers have existed since the first demonstration of a laser by Maiman. ${ }^{6}$ Lasers have operated in a number of states since that first demonstration. The complications introduced by pump fluctuations, spatial hole burning, inhomogeneous broadening, etc. have enhanced this variety of available states. The continuous-wave ring dye laser is a homogeneously broadened laser that contains few of these additional complications yet still displays a rich variety of operational states. Hillman et al. ${ }^{5}$ observed a continuous-wave dye laser, initially operating at a single frequency, go unstable and begin oscillating at two frequencies. As the pump power was increased, the two components of the laser spectrum separated with a linear dependence on the electric field strength of the laser. When the separation of the spectral components reached $\sim 80 \AA$, the laser encountered another instability threshold. The twofrequency state then collapsed to another two-frequency state of smaller frequency separation. When the pump power was increased still further, this new two-frequency state behaved similarly to the initial two-frequency state.

The physical mechanism for these new states of dye-laser operation is not well understood. There have been a number of theoretical treatments of a multimode laser. ${ }^{7-11} \mathrm{Un}$ fortunately, none of them has provided an explanation for all the features of the experimental results. The most successful theoretical model ${ }^{9-11}$ takes the molecular structure of the laser-dye molecule into account. However, the actual physical mechanism responsible for the instability remains to be seen.

In an attempt to develop physical intuition for the problem of a nonlinear system being driven by a two-component field, we previously examined the gain for such a field driving a two-level atom. ${ }^{8}$ These calculations showed that the gain of the two-component field displays resonances when the frequency separation of the two fields is equal to the Rabi frequency or to a subharmonic of the Rabi frequency.

In this paper we consider the stability of a bichromatic laser field. Since a general self-consistent solution to the multimode laser equations is not available, the stability analysis must be carried out in some other manner. The equations could be numerically integrated; however, the parameter space is too large for such a solution to provide intuition. An alternative is to calculate the atomic response to the laser field and examine the gain experienced by a probe field. When the probe-field gain exceeds the laserfield gain, an instability will occur. In this paper we discuss the stability of a bichromatic field to the growth of a bichromatic probe field by using just such an alternative.

The analytic description of the interaction of four field components with a resonant medium is a cumbersome task. The nonlinear response of the medium produces combination tones of the field components in the polarization of the medium. When the frequencies of the combination tones are incommensurate with one another, the medium can exhibit a broadband spectral response. ${ }^{12}$ The analytic description of such a problem becomes extremely difficult. However, if the frequencies of the combination tones are commensurate, one can employ Floquet's theorem ${ }^{13}$ to study the problem analytically. If dispersion in the cavity is neglected, the mode frequencies are all commensurate with one another. The mode frequencies are integer multiples of the inverse of the cavity round-trip time. Therefore the simplest physical situation is one in which the field frequencies are commensurate with one another. The most elementary four-component field is one in which each field component 


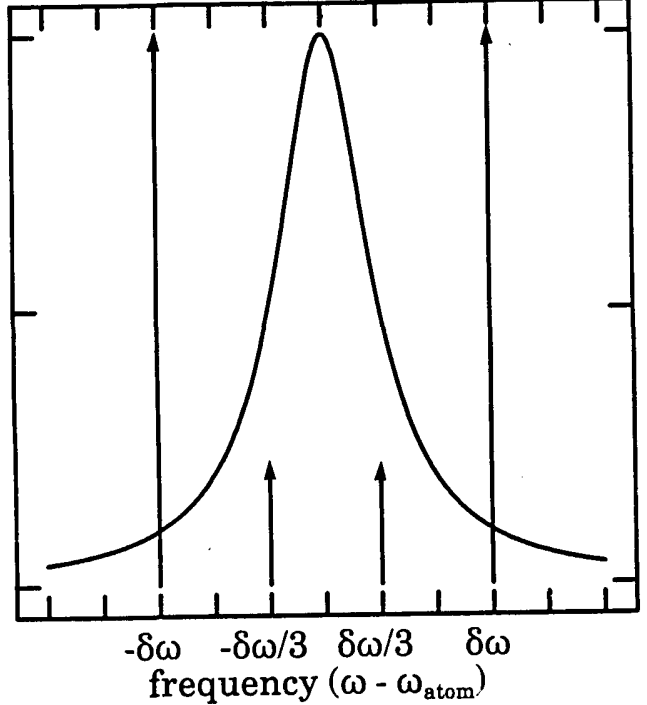

Fig. 1. Applied field spectrum. We plot the spectrum of the field used for the calculation as vertical arrows. The field is assumed to be symmetrically placed about the atomic resonance, $\omega_{\text {atom }}$. The atomic resonance is graphically displayed as a Lorentzian line shape. The field is made up of frequency components at $\omega-\delta \omega, \omega-$ $1 / 3 \delta \omega, \omega+1 / 3 \delta \omega$, and $\omega+\delta \dot{\omega}$. The components at $\omega+\delta \omega$ and $\omega-\delta \omega$ are referred to as the strong field in the text.

creates a degenerate beat frequency with the nearest other field component. We examine such a field and further specify the problem by considering only subharmonic probe fields. These probe fields are especially interesting, as they give us insight into possible physical mechanisms for subharmonic instabilities. The same analysis could be carried out for other subharmonic fields, such as the $1 / 2$ subharmonic observed in period-doubling sequences. The techniques used in the analysis of such a field would be identical; however, the dimension of the matrices used in the solution would be larger. The stability of bichromatic fields to harmonic bichromatic probe fields will be the subject of a future paper. ${ }^{14}$ For simplicity, the fields are symmetrically placed about the atomic resonance frequency; the extension of the problem to include asymmetric field states is straightforward. Figure 1 shows the spectrum of this symmetric field.

\section{OUTLINE OF THE CALCULATION}

The general expression for a four-component field is better understood by first analyzing the single $100 \%$ amplitudemodulated (AM) field. The expression for such a field is written as a two-component field or as a single component with a sinusoidal amplitude

$$
\begin{aligned}
E_{1}(t) & =2 \xi[\cos (\omega+\delta \omega) t+\cos (\omega-\delta \omega) t] \\
& =4 \xi \cos \delta \omega t \cos \omega t .
\end{aligned}
$$

The amplitude of each frequency component is equal to $2 \xi$.

The use of complex phasor diagrams to describe the phase relationships among multicomponent fields can be helpful. The complex phasor for a $100 \%$ AM field is a vector that oscillates along a line through the origin. The amplitude of the field determines the length of the vector; the modulation frequency determines the vector's rate of oscillation. In defining the field above, we chose an arbitrary phase factor so the complex phasor lies along the real axis of the complex plane.

There are two independent subharmonic probe fields at each modulation frequency. When the field is symmetric about the atomic resonance, as in our case, the independent probe fields are in phase or in quadrature to the strong field; these phasors will be along the real or imaginary axes, respectively. The expression for the $100 \%$ AM perturbative fields will be similar to the expression above; however, in general there will be a phase difference between the oscillations of the perturbative fields and the strong field. With these considerations in mind, we choose the perturbation to the $100 \%$ AM field for our calculations to be of the form

$$
\begin{aligned}
\delta E(t)= & 4 \delta \xi^{\prime} \cos \left(\frac{1}{3} \delta \omega t+\theta^{\prime}\right) \cos \omega t-4 \delta \xi^{\prime \prime} \\
& \times \sin \left(1 / 3 \delta \omega t+\theta^{\prime \prime}\right) \sin \omega t .
\end{aligned}
$$

In Eq. (2), the amplitude of the in-phase (in-quadrature) probe field is $\delta \xi^{\prime}\left(\delta \xi^{\prime \prime}\right)$. The phase between the oscillations of the strong field and the in-phase (in-quadrature) subharmonic probe field is $\theta^{\prime}\left(\theta^{\prime \prime}\right)$. The total field is the sum of Eqs. (1) and (2).

The optical Bloch equations model the response of a twolevel atom coherently driven by an optical field. ${ }^{14}$ In a reference frame rotating at the frequency $\omega$, the optical Bloch equations are

$$
\begin{aligned}
\dot{u} & =-\Omega^{\prime \prime}(t) w-u / T_{2}, \\
\dot{v} & =\Omega^{\prime}(t) w-v / T_{2}, \\
\dot{w} & =\Omega^{\prime \prime}(t) u-\Omega^{\prime}(t) v-\left(w-w_{\mathrm{eq}}\right) / T_{1},
\end{aligned}
$$

in which

$$
\begin{aligned}
\Omega^{\prime}(t) & =2 \kappa\left[\delta \xi^{\prime} \cos \left(\frac{1}{3} \delta \omega t+\theta^{\prime}\right)+\xi \cos \delta \omega t\right], \\
\Omega^{\prime \prime}(t) & =2 \kappa \delta \xi^{\prime \prime} \sin \left(1 / 3 \delta \omega t+\theta^{\prime \prime}\right),
\end{aligned}
$$

and $\kappa=2 d / \hbar$. The dipole-moment matrix element between the two levels is $d$, and $\hbar$ is Planck's constant. The slowly varying amplitude of the in-phase (in-quadrature) part of the dipole moment is $u(v)$. The inversion of the two-level system is $w ; w_{\mathrm{eq}}$ is the value to which the inversion decays in the absence of a coherent field. The relaxation times of the inversion and polarization are $T_{1}$ and $T_{2}$, respectively. The field components that are in phase (in quadrature) to the rotating frame are $\Omega^{\prime}(t)\left(\Omega^{\prime \prime}(t)\right)$.

Floquet's theorem tells us that in the stationary state the atomic variables will respond at harmonics of the drivingfield modulation frequency. So in the stationary state we express the atomic variables as Fourier series in the modulation frequency:

$$
\begin{gathered}
u=\sum_{n=-\infty}^{\infty} u_{n} \exp (1 / 3 i n \delta \omega t), \quad v=\sum_{n=-\infty}^{\infty} v_{n} \exp (1 / 3 i n \delta \omega t), \\
w=\sum_{n=-\infty}^{\infty} w_{n} \exp (1 / 3 i n \delta \omega t) .
\end{gathered}
$$

This assumption leads to the following recurrence relations: 


$$
\begin{aligned}
\left(1+1 / 3 i n \delta \omega T_{2}\right) u_{n}= & \left(\delta \Omega^{\prime \prime} w_{n-1}+\delta \Omega^{\prime \prime *} w_{n+1}\right) T_{2} \\
\left(1+\frac{1}{3} i n \delta \omega T_{2}\right) v_{n}= & \left(\delta \Omega^{\prime} w_{n-1}+\delta \Omega^{\prime *} w_{n+1}+\Omega^{\prime} w_{n-3}\right. \\
& \left.+\Omega^{\prime} w_{n+3}\right) T_{2}, \\
\left(1+1 / 3 i n \delta \omega T_{1}\right) w_{n}= & w_{\mathrm{eq}} \delta_{n, 0}-\left(\delta \Omega^{\prime \prime} u_{n-1}+\delta \Omega^{\prime \prime *} u_{n+1}\right. \\
& +\delta \Omega^{\prime} v_{n-1}+\delta \Omega^{\prime *} v_{n+1}+\Omega^{\prime} v_{n-3} \\
& \left.+\Omega^{\prime} v_{n+3}\right) T_{1},
\end{aligned}
$$

in which

$$
\begin{aligned}
\Omega^{\prime} & =\kappa \xi \\
\delta \Omega^{\prime} & =\kappa \delta \xi^{\prime} \exp \left(i \theta^{\prime}\right), \\
\delta \Omega^{\prime \prime} & =i \kappa \delta \xi \exp \left(i \theta^{\prime \prime}\right),
\end{aligned}
$$

and the asterisk denotes a complex conjugate. The Kronecker delta function is $\delta_{n, 0}$.

We solve for $u$ and $v$ in the first two recurrence relations and eliminate them from the third relation. The third relation then becomes a function of the Fourier components of $w$ alone:

$$
\begin{aligned}
a_{n} w_{n-6}+b_{n} w_{n-4}+c_{n} w_{n-2} & +d_{n} w_{n}+e_{n} w_{n+2} \\
& +f_{n} w_{n+4}+g_{n} w_{n+6}=w_{\text {eq }} \delta_{n, 0}
\end{aligned}
$$

in which

$$
\begin{aligned}
a_{n}= & L_{n-3} \Omega^{\prime 2} T_{1} T_{2}, \\
b_{n}= & \left(L_{n-1}+L_{n-3}\right) \Omega^{\prime} \delta \Omega^{\prime} T_{1} T_{2}, \\
c_{n}= & {\left[L_{n-1}\left(\delta \Omega^{\prime \prime 2}+\delta \Omega^{\prime 2}\right)+\left(L_{n+1}+L_{n-3}\right) \Omega^{\prime} \delta \Omega^{\prime *}\right] T_{1} T_{2}, } \\
d_{n}= & {\left[1+1 / 3 i n \delta \omega T_{1}+\left(L_{n+1}+L_{n-1}\right)\left(\left|\delta \Omega^{\prime \prime}\right|^{2}+\left|\delta \Omega^{\prime}\right|^{2}\right) T_{1} T_{2}\right.} \\
& \left.+\left(L_{n+3}+L_{n-3}\right) \Omega^{\prime 2} T_{1} T_{2}\right], \\
e_{n}= & {\left[L_{n+1}\left(\delta \Omega^{\prime \prime * 2}+\delta \Omega^{\prime * 2}\right)+\left(L_{n-1}+L_{n+3}\right) \Omega^{\prime} \delta \Omega^{\prime}\right] T_{1} T_{2}, } \\
f_{n}= & \left(L_{n+1}+L_{n+3}\right) \Omega^{\prime} \delta \Omega^{\prime *} T_{1} T_{2}, \\
g_{n}= & L_{n+3} \Omega^{\prime 2} T_{1} T_{2},
\end{aligned}
$$

and

$$
L_{n}=\left(1+1 / 3 i n \delta \omega T_{2}\right)^{-1} \text {. }
$$

This recurrence relation, Eq. (8), can be rewritten as a threeterm recurrence relation; the terms are products of matrixes and vectors:

$$
P_{n} W_{n-2}+Q_{n} W_{n}+R_{n} W_{n+2}=D_{n}
$$

in which

$$
\begin{aligned}
& P_{n}=\left[\begin{array}{ccc}
a_{3 n-2} & b_{3 n-2} & c_{3 n-2} \\
0 & a_{3 n} & b_{3 n} \\
0 & 0 & a_{3 n+2}
\end{array}\right], \\
& Q_{n}=\left[\begin{array}{ccc}
d_{3 n-2} & e_{3 n-2} & f_{3 n-2} \\
c_{3 n} & d_{3 n} & e_{3 n} \\
b_{3 n+2} & c_{3 n+2} & d_{3 n+2}
\end{array}\right],
\end{aligned}
$$

$$
\begin{gathered}
R_{n}=\left[\begin{array}{ccc}
g_{3 n-2} & 0 & 0 \\
f_{3 n} & g_{3 n} & 0 \\
e_{3 n+2} & f_{3 n+2} & g_{3 n+2}
\end{array}\right], \\
D_{n}=w_{\mathrm{eq}}\left[\begin{array}{c}
0 \\
\delta_{n, 0} \\
0
\end{array}\right], \quad W_{n}=\left[\begin{array}{c}
w_{3 n-2} \\
w_{3 n} \\
w_{3 n+2}
\end{array}\right] .
\end{gathered}
$$

We solve this three-term recurrence relation by using matrix continued fractions. ${ }^{15}$ The solution is given in Appendix A. Now that we have determined the atomic response, we calculate the gain experienced by the probe fields.

The time-averaged product of the probe field and the time derivative of the polarization gives the gain experienced by the probe field. The gain of the in-phase subharmonic probe field is proportional to the real part of the first harmonic of $v$ :

$$
\mathrm{d} \delta \xi^{\prime} / \mathrm{d} z=\alpha \operatorname{Re}\left[v_{1} \exp \left(-i \theta^{\prime}\right)\right] / 2 \kappa T_{2} .
$$

The gain of the in-quadrature subharmonic probe field is proportional to the imaginary part of the first harmonic of $u$ :

$$
\mathrm{d} \delta \xi^{\prime \prime} / \mathrm{d} z=\alpha \operatorname{Im}\left[u_{1} \exp \left(-i \theta^{\prime \prime}\right)\right] / 2 \kappa T_{2} .
$$

\section{DISCUSSION OF RESULTS}

In Fig. 2 we plot the in-phase subharmonic probe-field gain as a function of modulation frequency for various strongfield intensities. At low intensities the probe-field gain is a Lorentzian in modulation frequency with a width of $1 / T_{2}$. As the intensity of the strong field is increased, the gain curve develops a dip near zero modulation frequency. The dip has a width of $1 / T_{1}$. The presence of this dip implies that the probe field will experience the greatest gain for a nonzero modulation frequency. In Fig. 3 we plot the inquadrature subharmonic probe-field gain as a function of modulation frequency for various strong-field intensities. Again we see that at low intensities the probe-field gain is a Lorentzian in modulation frequency with a width of $1 / T_{2}$. As the strong-field intensity is increased, the gain curve develops an antidip near zero modulation frequency.

The calculations have shown both quadratures of the probe-field gain to be independent of $\theta^{\prime}$ and $\theta^{\prime \prime}$. In Appendix $B$ we show analytically that the in-quadrature subharmonic probe-field gain is independent of $\theta^{\prime \prime}$. The independence of subharmonic gain from the phase difference between the oscillations of the probe field and the oscillations of the strong field implies that the subharmonic component of the polarizations is directly proportional to the subharmonic field. In contrast, it can be shown that the harmonic probe-field gain exhibits a strong dependence on the phase difference between the strong field oscillations and the harmonic probe-field oscillations. Also, if the amplitude of the probe fields is increased, so that their interaction can no longer be considered only to first order, the gain of the subharmonic field becomes dependent on $\theta$. The independence of the gain from the phase implies that the instabilities predicted may be symmetry-breaking instabilities. 


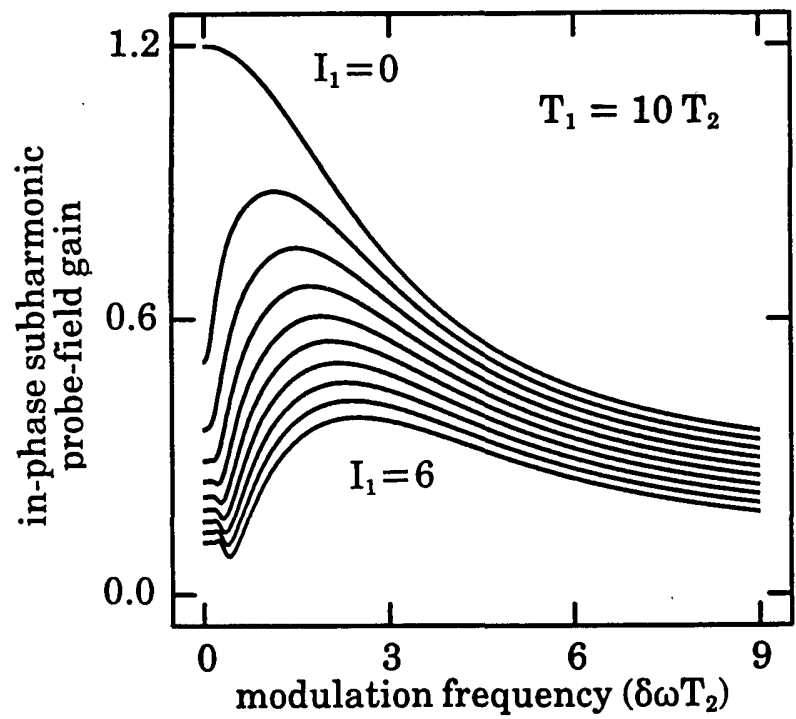

Fig. 2. In-phase subharmonic probe-field gain. We plot the inphase subharmonic probe-field gain as a function of $\delta \omega$ for various values of the strong-field intensity. A dip in the center of the gain curve develops at low intensity. This means that the maximum gain occurs for nonzero modulation frequencies. Time-averaged strong-field intensity, $I_{1}=2\left(\kappa \xi_{1}\right)^{2} T_{1} T_{2}=0,2 / 3,1 \frac{1}{3}, 2,2 \frac{2}{3}, 3 \frac{1}{3}, 4,4 \frac{2}{3}$, $5 \frac{1}{3}, 6$. Inversion decay time $T_{1}=10 T_{2}$.

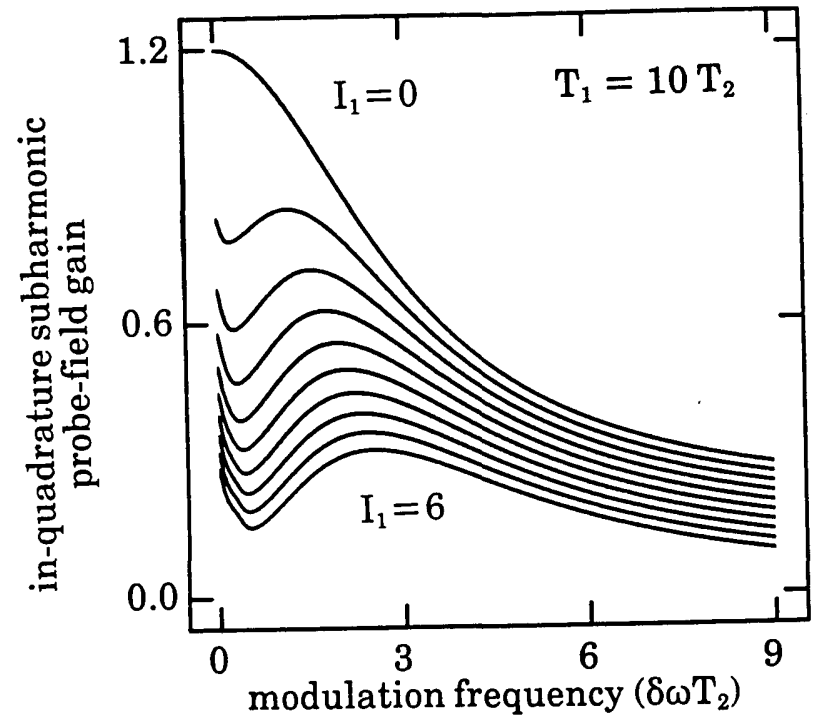

Fig. 3. In-quadrature subharmonic probe-field gain. We plot the in-quadrature subharmonic probe-field gain as a function of $\delta \omega$ for various values of the strong-field intensity. In contrast to the inphase subharmonic probe-field gain, the in-quadrature subharmonic probe-field gain develops an antidip near the center of the gain curve. The intensities and decay times are the same as for Fig. 2 .

The phase of the light field for a laser at the first laser threshold displays similar behavior. ${ }^{16}$

In Figs. 4 and 5, respectively, we show the in-phase and inquadrature subharmonic probe-field gains for larger strongfield intensities. In both cases, additional structure begins to develop about the initial feature. The additional maxima and minima seem to move out with a parabolic dependence on intensity.
In Figs. 6 and 7 we plot the peak position of the subharmonic gain curves as a function of modulation frequency and amplitude of the strong field. Here we can clearly see the peaks move out to higher frequencies linearly as the electric field increases. This linear dependence suggests a connection between these resonances and the Rabi frequency. In

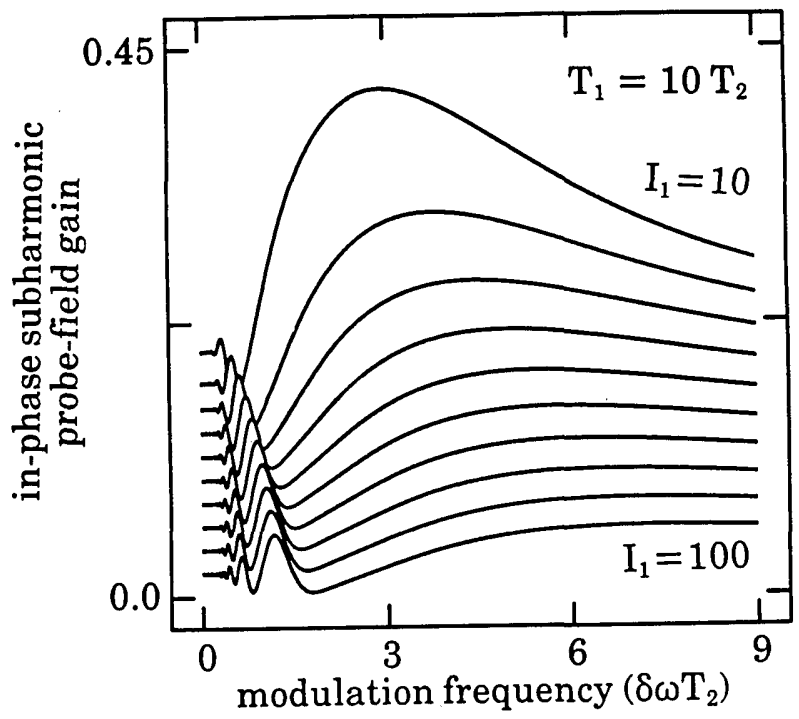

Fig. 4. In-phase subharmonic probe-field gain. We plot the inphase subharmonic probe-field gain as a function of $\delta \omega$ for larger values of the strong-field intensity. Structure develops within the dip that formed at low intensity. The additional structure appears at subharmonics of the main resonance. Time-averaged strong field intensity, $I_{1}=2\left(\kappa \xi_{1}\right)^{2} T_{1} T_{2}=10,20,30,40,50,60,70,80,90,100$. Inversion decay time $T_{1}=10 T_{2}$.

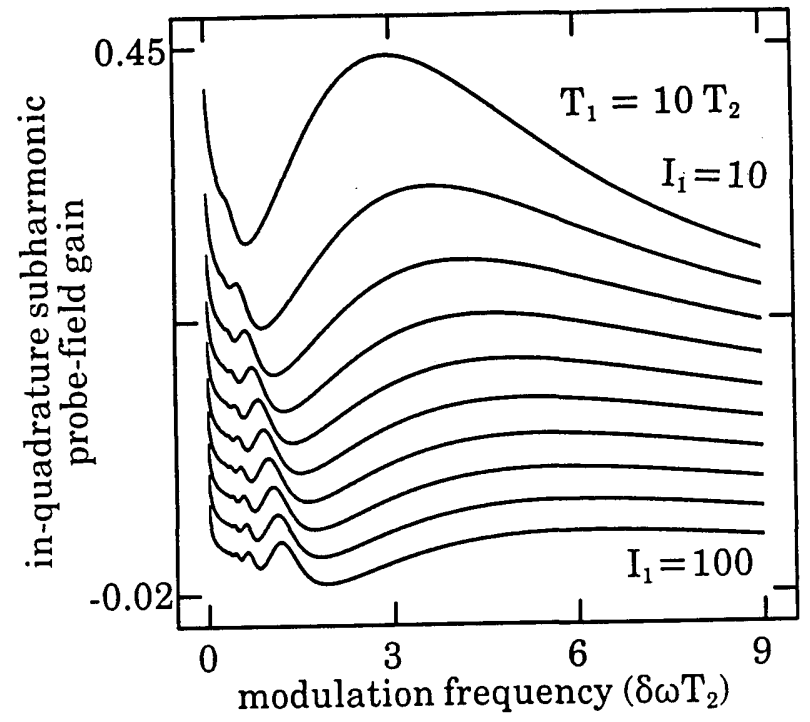

Fig. 5. In-quadrature subharmonic probe-field gain. We plot the in-quadrature subharmonic probe-field gain as a function of $\delta \omega$ for larger values of the strong-field intensity. Additional structure appears outside the antidip formed at low intensity. These peaks also appear at subharmonics of the main resonance. Time-averaged strong-field intensity, $I_{1}=2\left(\kappa \xi_{1}\right)^{2} T_{1} T_{2}=10,20,30,40,50,60$, $70,80,90,100$. Inversion decay time $T_{1}=10 T_{2}$. 


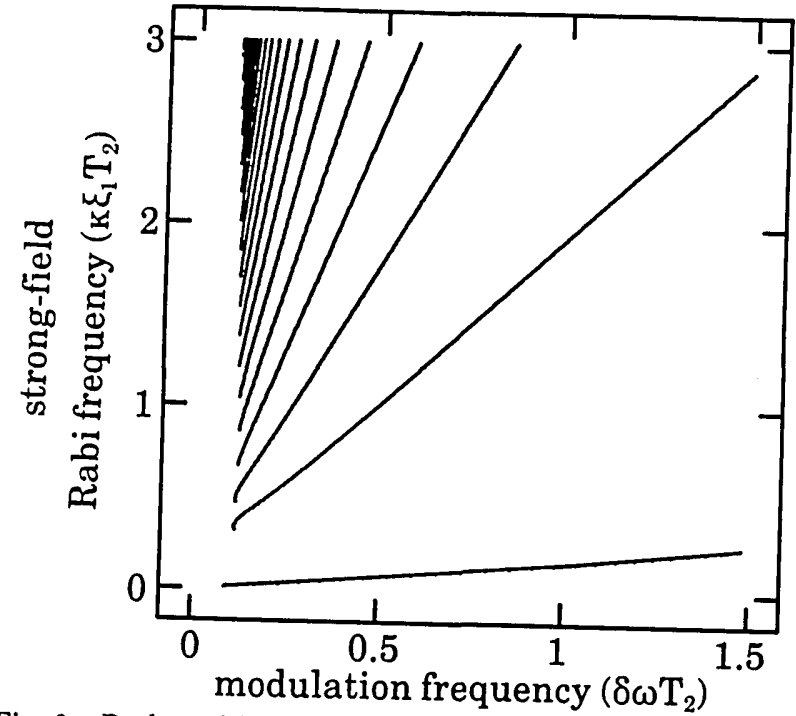

Fig. 6. Peak positions of the in-phase subharmonic probe-field gain. We plot the peak positions of the in-phase subharmonic probe-field gain as a function of Rabi frequency, $\kappa \xi_{1}$, and modulation frequency, $\delta \omega$. The position of the resonances in modulation frequency varies linearly with the strong-field Rabi frequency. Inversion decay time $T_{1}=10 T_{2}$.

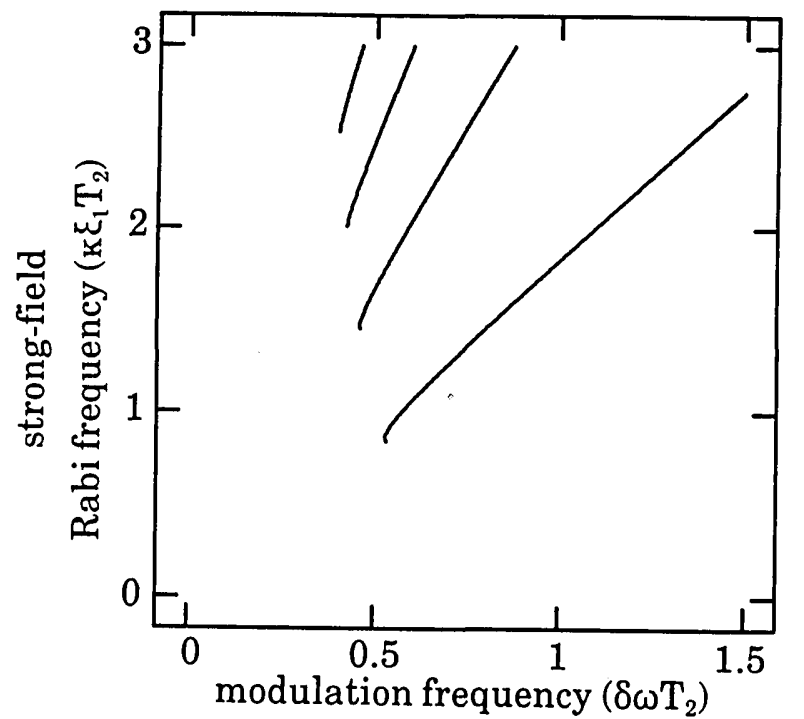

Fig. 7. Peak positions of the in-quadrature subharmonic probefield gain. We plot the peak positions of the in-quadrature subharmonic probe-field gain as a function of Rabi frequency, $\kappa \xi_{1}$, and modulation frequency, $\delta \omega$. The position in modulation frequency of the resonances of the in-quadrature subharmonic probe-field gain also varies linearly with strong-field Rabi frequency. The slopes of the lines are equal to the slopes of the lines in Fig. 6. The decay times are the same as in Fig. 6.

contrast to the two-field problem, where the positions of the resonances are found to be independent of the damping rates, ${ }^{8}$ the positions of the subharmonic probe-field resonances do display a dependence on damping. The maxima occurring at the largest modulation frequency vary most drastically with variation of the ratio between $T_{1}$ and $T_{2}$. The subsequent peaks vary less and less sensitively. The physical mechanism responsible for this behavior is not well understood. Some of the features, however, are similar in behavior to that seen in multiphoton absorption. ${ }^{17}$ Subhar- monic resonances such as these are also present in the twofrequency problem and have also been predicted in other multichromatic analyses. ${ }^{17}$

In Fig. 8 we show the strong-field gain as a function of modulation frequency for a fixed strong-field amplitude. In Ref. 8 the maxima in the strong-field gain curve were found to occur where the zeroth-order Bessel function, $J_{0}(2 \kappa \xi / \delta \omega)$, is equal to zero. For large Rabi frequencies, $\kappa \xi$, and small modulation frequencies, $\delta \omega$, the zeros of the Bessel function become equally spaced. It is in these regimes that the resonances become subharmonics. In Fig. 9 we plot the in-

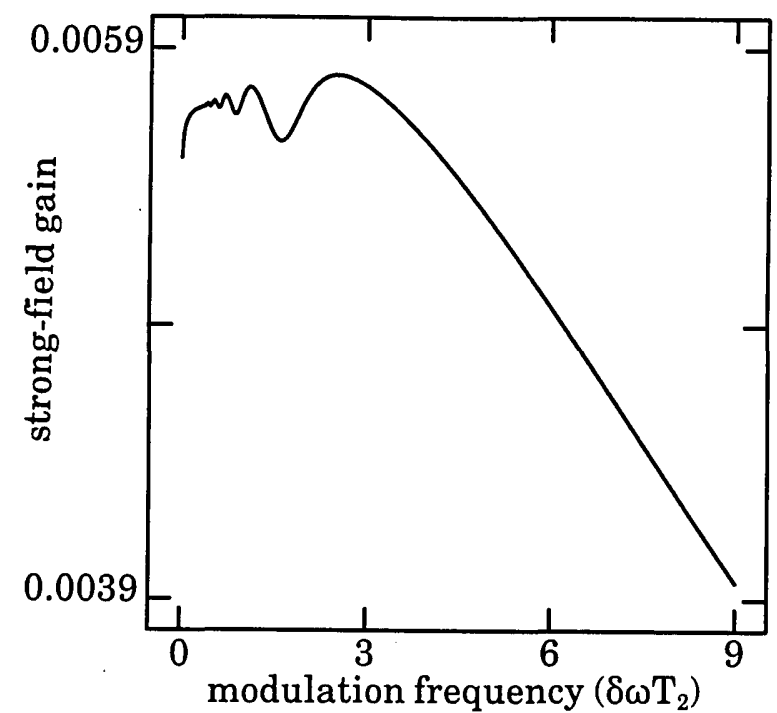

Fig. 8. Strong-field gain curve. We plot the strong-field gain as a function of modulation frequency, $\delta \omega$. The maxima occur at the zeros of the zeroth-order Bessel function, $J_{0}\left(2 \kappa \xi_{1} / \delta \omega\right)$. Rabi frequency of the strong field, $\kappa \xi_{1}=5 / T_{2}$. Inversion decay time $T_{1}=$ $10 T_{2}$.

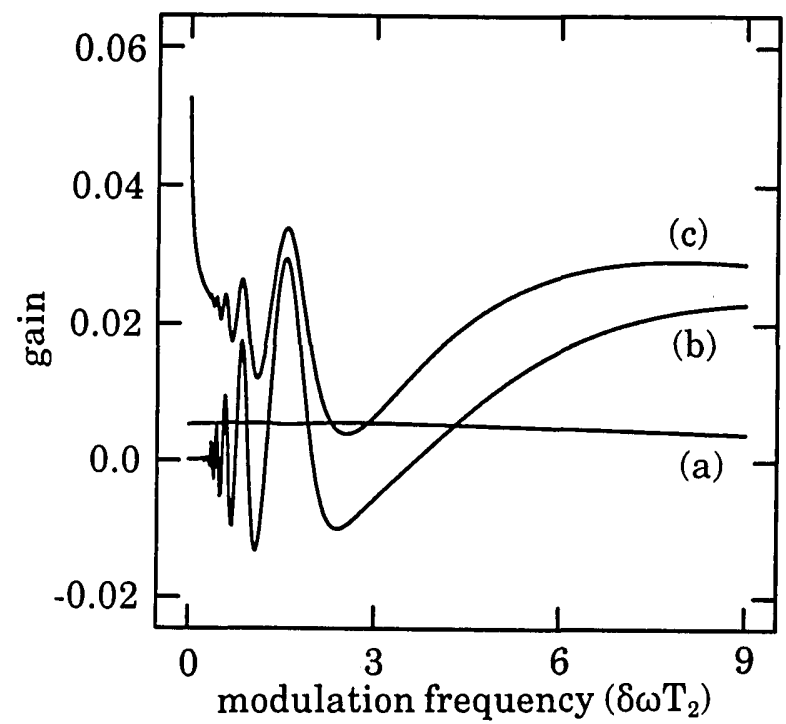

Fig. 9. Gain curve of the three pairs of fields. We plot (a) the strong-field gain, (b) the in-phase subharmonic probe-field gain, and (c) the in-quadrature subharmonic probe-field gain as a function of modulation frequency, $\delta \omega$, for the same parameters as in Fig. 8. The absolute maxima of the strong-field gain curve exceeds the subharmonic probe-field gains. At that modulation frequency the strong field will be stable to the growth of subharmonic probe fields. 


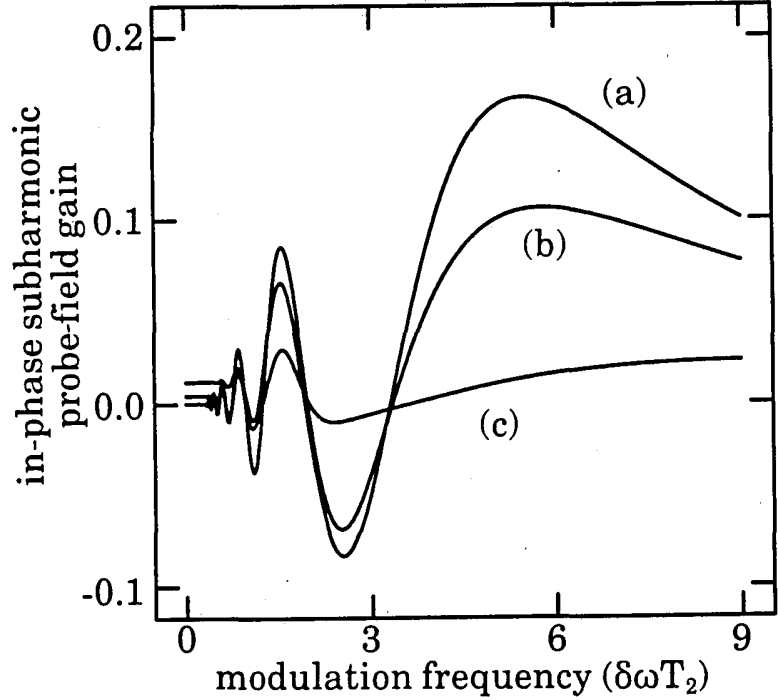

Fig. 10. Influence of damping on the in-phase subharmonic probefield gain. We plot the in-phase subharmonic probe-field gain as a function of modulation frequency for three different ratios of $T_{1}$ to $T_{2}$. Rabi frequency of the strong field, $\kappa \xi_{1}=3 / T_{2}$. The inversion decay times for the three curves are (a) $T_{1}=1 / 2 T_{2}$, (b) $T_{1}=T_{2}$, and (c) $T_{1}=10 T_{2}$.

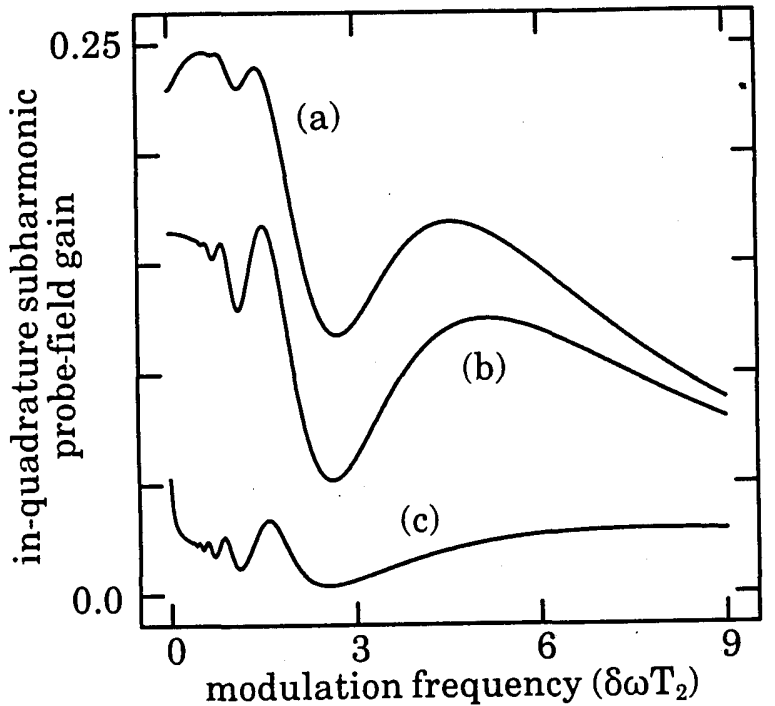

Fig. 11. Influence of damping on the in-quadrature subharmonic probe-field gain. We plot the in-quadrature subharmonic probefield gain as a function of modulation frequency for three different ratios of $T_{1}$ to $T_{2}$. Rabi frequency of the strong field, $\kappa \xi_{1}=3 / T_{2}$. The inversion decay times for the three curves are (a) $T_{1}=1 / 2 T_{2}$, (b) $T_{1}=T_{2}$, and (c) $T_{1}=10 T_{2}$.

phase, in-quadrature, and strong-field gains as a function of modulation frequency for the same parameters as for Fig. 8 . At this intensity the in-quadrature probe-field gain is always positive; it exceeds the strong-field gain for all modulation frequencies, except in the vicinity of the absolute maxima of the strong-field gain curve. The in-phase probe-field gain takes on both positive and negative values as a function of modulation frequency. It can also be noted that the oscillations in modulation frequency of the two subharmonic gain curves are $180^{\circ}$ out of phase with the oscillations in modulation frequency of the strong-field gain. It is important to remember, however, that the frequency separation of the probe fields is one third the modulation frequency, $\delta \omega$. This means that the presence of a frequency-dependent loss influences the two curves differently. For instance, a frequency-dependent loss that increases with increasing modulation frequency reduces the strong-field gain curve more than it does the probe-field gain curve. This type of frequencydependent loss models the frequency-selection elements used in lasers. Reduction of the strong-field gain makes it possible for the probe-field gain to exceed the strong-field gain. If the strong field is the field in a laser, this reduction allows the probe field sufficient gain to overcome losses and begin oscillation. The severity of the frequency-dependent loss determines the point at which the instability will occur. The experiments of Hillman et al..$^{5}$ and Stroud et al. ${ }^{18}$ confirm these conclusions. A high-dispersion flint-glass prism was used as the frequency-selection element by Hillman et al. ${ }^{5}$ They found that the bichromatic field became unstable after a splitting of $\sim 80 \AA$. The 80 - $\AA$ bichromatic field collapsed to a bichromatic field of smaller separation. A lowdispersion quartz-glass prism was used by Stroud et al:, ${ }^{18}$ and the bichromatic field was found to remain stable to a separation of $340 \AA$. While the frequency-dependent loss of the quartz-glass prism was insufficient to destabilize the bichromatic field, the dispersion of the flint-glass prism provided sufficient frequency-dependent loss to destabilize the bichromatic field at a splitting of $\sim 80 \AA$.

In Figs. 10 and 11 we plot a series of in-phase and inquadrature subharmonic probe-field gain curves for fixed strong-field amplitudes as a function of modulation frequency and three different ratios of damping times. The ratios chosen are indicative of collisional dephasing $\left(T_{2} \ll T_{1}\right)$, strong collisions $\left(T_{2}=T_{1}\right)$, and radiative broadening $\left(T_{2}=\right.$ $\left.2 T_{1}\right)$. These plots indicate that the shape of the in-phase gain curve is not a strong function of the ratio of damping times. However, as noted above, the positions of the maxima do show a dependence on the ratio of $T_{1}$ to $T_{2}$. The inquadrature subharmonic probe-field gain does indicate a dependence on the ratio of $T_{1}$ and $T_{2}$ for modulation frequencies less than $1 / T_{2}$. However, for modulation frequencies greater than the linewidth, $1 / T_{2}$, the curves are quite similar in form and show only a weak dependence on the ratio of $T_{1}$ to $T_{2}$.

\section{CONCLUSIONS}

We have calculated the atomic response to a pair of $100 \%$ AM fields. We used this solution to calculate the gain seen by a $100 \%$ AM subharmonic probe field. The subharmonic probe field sees a Lorentzian line shape in modulation frequency for the limit of small amplitude. As the strong field amplitude is increased, it modifies the line shape experienced by the subharmonic probe field. The line shape contains a primary resonance and subharmonic resonances of this primary resonance. The positions of the resonances vary linearly with the Rabi frequency of the strong field. The locations of the resonances also depend on the ratio of the inversion decay rate to the dipole decay rate.

We have also observed that the gain of the subharmonic probe field is independent of the phase between the oscillations of the strong field and the oscillations of the subharmonic probe field. This independence of phase implies that 
the subharmonic response of the atom is directly proportional to the subharmonic field. It also predicts that the instabilities will be independent of this phase difference.

The calculations also reveal that the $100 \%$ AM strong field is stable to the growth of subharmonic fields if there are no frequency-dependent loss mechanisms. However, if frequency-dependent loss mechanisms are present, we predict that the $100 \%$ AM field will go unstable. The point at which the field goes unstable depends on the severity of the frequency-dependent loss. This conclusion agrees well with observed instabilities in cw dye lasers. ${ }^{5,18}$

\section{APPENDIX A}

In this appendix we outline the method for calculating the gain of the subharmonic probe fields.

The larger harmonic components of $w$ must become less significant if the Fourier series in Eqs. (5) are to converge and the rotating-wave approximation is to remain valid. Therefore we assume that the harmonic components of the inversion, $w_{n}$, are negligible for $n>N$. With this assumption, the recurrence relation, Eq. (10), for $n=N$ can be written as

$$
P_{N} W_{N-2}+Q_{N} W_{N}=0,
$$

which implies that

$$
W_{N}=-Q_{N}^{-1} P_{N} W_{N-2} \text {. }
$$

The recurrence relation, Eq. (10), for $n=N-2$ can now be written as

$$
P_{N-2} W_{N-4}+\left(Q_{N-2}-R_{N-2} Q_{N}^{-1} P_{N}\right) W_{N-2}=0,
$$

which implies that

$$
W_{N-2}=-\left(Q_{N-2}-R_{N-2} Q_{N}{ }^{-1} P_{N}\right)^{-1} P_{N-2} W_{N-4} .
$$

We continue in such a fashion, defining the larger-index Fourier amplitudes in terms of the lower-index Fourier amplitudes, until we reach the relation for $n=2$. This relation has the following form:

$$
\begin{aligned}
W_{2}= & -\left(Q_{2}-R_{2}\left(Q_{4}-R_{4}\left(\ldots \left(Q_{N-2}-R_{N-2} Q_{N}\left({ }^{-1} P_{N}\right)^{-1}\right.\right.\right.\right. \\
& \left.\left.\left.\left.\times P_{N-2}\right)^{-1} P_{N-4} \ldots\right)^{-1} P_{6}\right)^{-1} P_{4}\right)^{-1} P_{2} W_{0}
\end{aligned}
$$

or, more simply,

$$
W_{2}=M W_{0},
$$

where $M$ is the matrix continued fraction, with elements $m_{i j}$. This expression, Eq. (A6), relates the harmonic components $w_{4}, w_{6}$, and $w_{8}$ to the harmonic components $w_{0}, w_{2}$, and $w_{-2}$. Strictly speaking, the matrix $M$ is a function of $N$. However, $N$ is chosen sufficiently large that $M$ becomes independent of $N$ to the desired accuracy of the calculation. This is our criterion of convergence. Since the inversion, $w$, is a real quantity, the Fourier components obey the relation

$$
w_{n}=w_{-n}{ }^{*} .
$$

We now consider Eq. (8) for the cases $n=-4,-2,0,2,4$. Using Eqs. (A6) and (A7), we write these five relations in matrix form as

$$
S W=W_{0},
$$

where the vectors $W$ and $W_{0}$ are

$$
W=\left[\begin{array}{l}
w_{4}^{*} \\
w_{2}{ }^{*} \\
w_{0} \\
w_{2} \\
w_{4}
\end{array}\right], \quad W_{0}=\left[\begin{array}{l}
0 \\
0 \\
w_{\text {eq }} \\
0 \\
0
\end{array}\right] .
$$

The matrix $S$ has elements $s_{i j}$, where

$$
\begin{aligned}
& s_{11}=d_{-4}+a_{-4} m_{33} *+b_{-4} m_{23} *+c_{-4} m_{13} * \text {, } \\
& s_{12}=e_{-4}+a_{-4} m_{32}{ }^{*}+b_{-4} m_{22}{ }^{*}+c_{-4} m_{12}{ }^{*} \text {, } \\
& s_{13}=f_{-4}+a_{-4} m_{31} *+b_{-4} m_{21} *+c_{-4} m_{11} * \text {, } \\
& s_{14}=g_{-4} \text {, } \\
& s_{15}=0 \text {, } \\
& s_{21}=c_{-2}+a_{-2} m_{23} *+b_{-2} m_{13} * \text {, } \\
& s_{22}=d_{-2}+a_{-2} m_{22}{ }^{*}+b_{-2} m_{12}{ }^{*} \text {, } \\
& s_{23}=e_{-2}+a_{-2} m_{21} *+b_{-2} m_{11}{ }^{*} \text {, } \\
& s_{24}=f_{-2} \text {, } \\
& s_{25}=g_{-2}, \\
& s_{31}=b_{0}+a_{0} m_{13}{ }^{*} \\
& s_{32}=c_{0}+a_{0} m_{12}^{*} \text {, } \\
& s_{33}=d_{0}+a_{0} m_{11} *+g_{0} m_{11} \text {, } \\
& s_{34}=s_{32}{ }^{*} \text {, } \\
& s_{35}=s_{31}{ }^{*} \text {, } \\
& s_{41}=a_{2}, \\
& s_{42}=b_{2} \text {, } \\
& s_{43}=c_{2}+f_{2} m_{11}+g_{2} m_{21} \text {, } \\
& s_{44}=d_{2}+f_{2} m_{12}+g_{2} m_{22} \text {, } \\
& s_{45}=e_{2}+f_{2} m_{13}+g_{2} m_{23} \text {, } \\
& s_{51}=0 \text {, } \\
& s_{52}=a_{4}, \\
& s_{53}=b_{4}+e_{4} m_{11}+f_{4} m_{21}+g_{4} m_{31} \text {, } \\
& s_{54}=c_{4}+e_{4} m_{12}+f_{4} m_{22}+g_{4} m_{32} \text {, } \\
& s_{55}=d_{4}+e_{4} m_{13}+f_{4} m_{23}+g_{4} m_{33} \text {. }
\end{aligned}
$$

Equation (A8) represents a system of five linear equations with five unknowns. The solution gives us expressions for $w_{0}, w_{2}, w_{4}$, and their complex conjugates. We can use these expressions to find the other Fourier components of $w$. From Eqs. (6a) and (6b) we can then determine the Fourier components of $u$ and $v$ that determine the gain. 


\section{APPENDIX B}

In this appendix we show that the gain of the in-quadrature subharmonic probe field is independent of the phase difference, $\theta^{\prime \prime}$, between the oscillations of the subharmonic probe field and the strong $100 \%$ AM field.

The solution for the atomic response to a single resonant $100 \%$ AM field has been found in Ref. 8. If we linearize the Bloch equations about this solution, the equation of motion for the perturbation to the slowly varying amplitude of the in-phase dipole moment becomes

$$
\delta \dot{u}=-\delta \Omega^{\prime \prime}(t) w_{\mathrm{AM}}-\delta u / T_{2},
$$

where $\delta u$ is the perturbation to the slowly varying amplitude of the in-phase dipole moment. The perturbative field in quadrature to the $100 \% \mathrm{AM}$ field is $\delta \Omega^{\prime \prime}(t)$. The stationary solution for the inversion to the Bloch equations driven by a resonant $100 \% \mathrm{AM}$ field is $w_{\mathrm{AM}}$. Recall that $w_{\mathrm{AM}}$ can be written as

$$
w_{\mathrm{AM}}=\sum_{n=-\infty}^{\infty} w_{n} \exp (i n \delta \omega t)
$$

If $\delta \Omega^{\prime \prime}(t)=2 \delta \Omega^{\prime \prime} \sin \left(1 / 3 \omega t+\theta^{\prime \prime}\right)$ we can employ Floquet's theorem and write the stationary solution to Eq. (B1) as

$$
\delta u=\sum_{n=-\infty}^{\infty} \delta u_{n} \exp (1 / 3 i n \delta \omega t)
$$

This assumption leads to the following recurrence relation:

$$
\begin{aligned}
\left(1+1 / 3 i n \delta \omega T_{2}\right) \delta u_{n}=i \delta \Omega^{\prime \prime} T_{2} & {\left[w_{(n-1) / 3} \exp \left(i \theta^{\prime \prime}\right)\right.} \\
& \left.-w_{(n+1) / 3} \exp \left(-i \theta^{\prime \prime}\right)\right] .
\end{aligned}
$$

The gain of the in-quadrature subharmonic probe field is proportional to $\delta u_{1} \exp \left(-i \theta^{\prime \prime}\right)$. From Eq. (B4) we find

$$
\delta u_{1} \exp \left(-i \theta^{\prime \prime}\right)=i \delta \Omega^{\prime \prime} T_{2} w_{0} /\left(1+1 / 3 i \delta \omega T_{2}\right) .
$$

The right-hand side of Eq. (B5) is independent of $\theta^{\prime \prime}$; this implies that the in-quadrature subharmonic probe-field gain is independent of the phase difference between the oscillations of the in-quadrature probe field and the oscillations of the strong field.

\section{ACKNOWLEDGMENTS}

We thank the U.S. Army Research Office-University Research Initiative for financial support of this research.

\section{REFERENCES}

1. N. B. Abraham, L. A. Lugiato, and L. M. Narducci, J. Opt. Soc. Am. B 2, 7 (1985). This issue of the journal is a special issue on instabilities in optically active media.

2. D. K. Bandy, A. N. Oraevsky, and J. R. Tredicce, eds., J. Opt. Soc. Am. B 5, 879-1215 (1988). This issue of the journal is a special issue on laser instabilities.

3. C. O. Weiss and J. Brock, Phys. Rev. Lett. 57, 2804 (1986).

4. R. S. Gioggia and N. B. Abraham, Phys. Rev. Lett. 51, 650 (1983).

5. L. W. Hillman, J. Krasinski, R. W. Boyd, and C. R. Stroud, Jr., Phys. Rev. Lett. 52, 1605 (1984).

6. T. H. Maiman, Nature 187, 493 (1960).

7. L. A. Lugiato, L. M. Narducci, E. V. Eschenazi, D. K. Bandy, and N. B. Abraham, Phys. Rev. A 32, 1563 (1985).

8. L. W. Hillman, J. Krasinski, K. Koch, and C. R. Stroud, Jr., J. Opt. Soc. Am. B 2, 211 (1985).

9. H. Fu and H. Haken, Phys. Rev. A 36, 4802 (1987).

10. H. Fu and H. Haken, J. Opt. Soc. Am. B 5, 899 (1988).

11. H. Fu and H. Haken, Phys. Rev. Lett. 60, 2614 (1988).

12. Y. Pomeau, B. Dorizzi, and B. Grammaticos, Phys. Rev. Lett. 56, 681 (1986).

13. E. T. Whittaker and G. N. Watson, Modern Analysis (Cambridge U. Press, Cambridge, 1965).

14. L. Allen and J. Eberly, Optical Resonance and Two-Level Atoms (Wiley, New York, 1975).

15. H. Risken and H. D. Vollmer, Z. Phys. B 39, 339 (1980).

16. H. Haken, Synergetics (Springer-Verlag, Berlin, 1983).

17. G. S. Agarwal and N. Nayak, J. Phys. B 19, 3385 (1986).

18. C. R. Stroud, Jr., K. Koch, and S. H. Chakmakjian, in Optical Instabilities, R. W. Boyd, M. G. Raymer, and L. M. Narducci, eds. (Cambridge U. Press, Cambridge, 1986), pp. 274-276. 